\title{
A Review of the Role of Interleukin-2 in the Pathophysiology of Major Depressive Disorder in Hospitalized Patients
}

\author{
Seyed Alireza Seyed Ebrahimi ${ }^{\circledR}$, Elham Karamian², Zahra Sadat Goli ${ }^{\circledR}$, Leila Sadat Mirseifi ${ }^{3^{*}}$ \\ 'Department of General Psychology, Faculty of Humanities, Badrud Branch, Islamic Azad University, Isfahan, Iran \\ ${ }^{2}$ Department of General Psychology, Faculty of Humanities, Kashan Branch, Islamic Azad University, Isfahan, Iran \\ ${ }^{3}$ Islamic Azad University, Kish Branch, Bushehr, Iran
}

*Corresponding Author: Leila Sadat Mirseifi, Ph.D. Candidate of Health Psychology, Islamic Azad University, Kish Branch, Bushehr, Iran. Tel: +98-9134366799, Email: leilamirseifi@gmail.com

Received April 12, 2021; Accepted June 27, 2021; Online Published September 12, 2021

\begin{abstract}
Background: Hospitalization due to any reason or medical condition is associated with fear, anxiety, and depression. Psychological and physiological factors have a significant impact on hospitalization outcomes.

Objectives: Given the functional importance of inflammatory cytokines and studies in previous studies on the relationship between inflammatory cytokines and major depressive disorder, we will focus more on studies on the role of interleukin 2 (IL-2) in the pathophysiology of major depressive disorder in hospitalized patients.

Methods: We used PubMed, Scopus, and Elsevier databases to search for articles from 1999 to 2021, emphasizing the studies of the last five years.

Results: In general, there was no consistent pattern in the observed relationships between cytokine concentrations or changes and clinical signs of significant depression. IL-2 and IL, two receptors in the body, play an essential role in the treatment and the pathophysiology of depression and major depression.

Conclusion: Finally, it can be concluded that hospitalization generally exposes the patient to inflammation. Studies show an increased risk of inflammation following hospitalization of patients, and many studies confirm the association of major depression with inflammatory cytokines and, more concentrated, IL-2.

Keywords: Interleukin 2, Depressive Disorder, Hospitalization, Depression, Anxiety, Inflammation
\end{abstract}

\section{Background}

Depression is one of the current problems in advanced and developing societies. ${ }^{1}$ Studies show that depression is a psychiatric disorder and leads to various physical problems. ${ }^{2}$ Immune system disorders are one of the most important problems that depression causes. ${ }^{3}$ Studies show that depression, on the one hand, suppresses the appropriate response to infectious agents by inducing the responses of type 2 helper T lymphocytes (responses involved in causing allergies), and some antibody-dependent autoimmunity, and on the other hand, causes chronic and destructive inflammation by the immune system. ${ }^{4}$ Inflammation itself exacerbates depression. Chronic inflammation is one of the physical problems caused by depression. ${ }^{5}$

On the other hand, researchers believe that chronic inflammation can be one of the causes of depression. ${ }^{6}$ Depression is a mental disorder that affects 15 million people a year. Depression is a disorder characterized by mood swings and includes feelings of sadness ranging from mild frustration to severe despair. ${ }^{7}$ This mood swing is characterized by a person's behavior, performance, and physiological actions. From a cognitive point of view, depression occurs among people who have negative selfesteem, show harmful assessments, and have a pessimistic attributional style. ${ }^{8}$

Conversely, a healthy person is mentally happy, physically healthy, politically aware, and culturally aware. ${ }^{9}$ In immunological responses, several molecules are involved in causing inflammation, especially chronic inflammation. So that, it can be linked to the Janus kinase/signal (JAK/ STAT) pathways that transcription transducers and transcription activators seek stimulation by cytokines. He pointed to inflammation following induction by microbial sensors such as absent in melanoma II, Toll-like receptor, node-like receptor (NLR), and AIMII. ${ }^{10,11}$ Studies show that several molecules are involved in causing inflammation in

Copyright (C 2021 The Author(s). This is an open-access article distributed under the terms of the Creative Commons Attribution License (http:// creativecommons.org/licenses/by/4.0), which permits unrestricted use, distribution, and reproduction in any medium, provided the original work is properly cited. 
patients with depression, the most important of which are inflammatory cytokines. ${ }^{12}$ Therefore, studying the pattern of cytokines and the pathways that lead to the production of inflammatory cytokines in patients with depression can help determine the pathogenesis of the disease. ${ }^{13}$ Receptorlike molecules, inflammatory inducers, NLR molecules, and signaling molecules are among the most important molecules that initiate inflammatory responses that increase cytokine expression. ${ }^{14}$

\section{Objectives}

Given the functional importance of inflammatory cytokines and previous studies on the relationship between inflammatory cytokines and major depressive disorder, we will focus more on studies on interleukin 2 (IL-2) in the pathophysiology of major depressive disorder in hospitalized patients.

\section{Methods}

In this review study, PubMed, Scopus, and Elsevier databases searched for articles from 1999 to 2021, emphasizing the studies of the last five years. Keywords used included interleukin 2, depressive disorder, hospitalization, depression, anxiety, inflammation. The number of articles found with these keywords was 50, from which 20 articles were selected, and their results were reviewed.

\section{Results}

\subsection{Major Depression}

Major depression is not just a temporary feeling of sadness, grief, or nostalgia. ${ }^{15}$ Depression is a condition beyond being sad or very sad about the loss of a loved one. Sadness and grief are natural and temporary reactions to the stresses of life. ${ }^{16}$ These moods go away in a relatively short time, and the person regains his normal state. Depression often referred to as clinical depression, mood disorder, or emotional disorder, can last for months or even years. ${ }^{17}$ The depressive illness affects a person's emotions, thoughts, behavior, and physical health. Depression is a mental illness. ${ }^{18}$ Almost everyone feels at least slightly depressed. Feelings of sadness, boredom, sadness, hopelessness, discouragement, and dissatisfaction are all common experiences of depression. ${ }^{19}$ However, familiarity with these conditions does not lead to awareness and knowledge because only in the last thirty years, significant progress has been made in recognizing this disease. ${ }^{20}$ In everyday language, depression refers to an emotional state, reaction to a situation, and a specific behavior style. ${ }^{21}$ Feelings of depression are often referred to as grief and may occur in rainy weather, biting cold, or after a fight with a friend. ${ }^{22} \mathrm{~A}$ situation that is expected to be happy puts an end to such a feeling of sadness. For example, people may feel sad after the New Year holidays, after moving to a new home, or after the baby is born. ${ }^{23}$ Defining depression is not easy. It is more difficult to classify, and it seems impossible to suggest a plan accepted by all experts and researchers. ${ }^{24}$

In general, the concept of depression has been used in three different ways. ${ }^{25}$ First, to identify normal feelings of sadness, despair, hopelessness, and their occurrence as a sign of a disorder, to identify depressive feelings in the context of mental disorders are some of the causes and types of mobility and respond to some therapies. ${ }^{26}$ Second, give a brief description of a symptom that includes the emotional, cognitive, motor, physiological, and secretory glands. Multiple definitions of depression refer to multiple symptoms combined in different ways. Sometimes, it is very difficult to recognize depression as a disorder of these compounds, especially if it has a hidden aspect or physical manifestations. ${ }^{27}$ Take yourself. However, it can be accepted that depression is also associated with physical and psychological symptoms, and physical symptoms sometimes overshadow the clinical table to such an extent that it prevents the recognition of depression. ${ }^{28}$

Because depression is a complex disease with heterogeneous symptoms, it is not always easy to diagnose. ${ }^{29,30}$ Many depressed patients do not complain of dysphoric mood. Their predominant clinical features are anxiety, physical complaints, fatigue, and genital symptoms such as changes in sleep and appetite or psychosis. ${ }^{31,32}$ Nevertheless, the physical symptoms of depression are less noticeable. The prevalence of this disorder in medical and surgical departments is 2 to 3 times higher than its prevalence in the general population. 10\%-30\% of short-term inpatient and surgical ward patients with psychiatric or psychosocial disorders and depressed patients in medical wards use medical resources three times more than non-depressed patients and double the cost seven times. They mostly go to emergency centers. ${ }^{32}$ Unfortunately, depression also negatively affects the course of illness, increasing the length of hospital stays. In a study in the medical and surgical wards, the length of hospital stay of depressed patients was on average ten days longer than non-depressed patients. ${ }^{33}$ In the same study, the group of depressed patients treated with antidepressants or psychotherapy had an average hospital stay of 8.31 days less than those who had not been treated. ${ }^{34}$ Numerous studies have shown the effect of depression on the course and outcome of medical and surgical diseases. Also, in most studies, treatment with antidepressants has been associated with better results. ${ }^{35,36}$ Today, surgeons are aware of the dimensions of their patients' psychiatric care. Such care is necessary both before and after surgery. Severe anxiety and preoperative depression greatly impact accepting surgery, hospitalization, and cooperation. ${ }^{37}$ After the hospitalization, depression causes the patient to be unmotivated and uninterested and reduces cooperation with the treatment, thus delaying recovery and prolonging hospitalization. ${ }^{38}$ Hospitalization is a potentially healing incident associated with fear, anxiety, and depression, and psychological and psychological factors significantly impact its outcome. ${ }^{39}$

The major depressive disorder may occur alone or be part of bipolar disorder. If it occurs alone, it is also called unipolar depression. ${ }^{40}$ Symptoms should persist for 
at least two weeks and indicate a change in the patient's past performance. It is twice as common in men as in women. Detective events occur in at least $25 \%$ of cases. Symptoms of the disorder change throughout the day and are more severe in the early morning. ${ }^{41}$ There is mental retardation or restlessness associated with vegetative symptoms. Delusions and hallucinations may occur with mood. The average age of onset of the disorder is 40 , but it can occur at any age. ${ }^{42}$ Genetic factors play a role. ${ }^{36}$ Most people who suffer from depression do not have periods of mania. However, one in two depressed patients develops both periods of depression and mania and is diagnosed with bipolar disorder or depressive mania. ${ }^{43}$ In this type of disorder, a person fluctuates between periods of depression and severe euphoria. In some hospitalized patients, only a short normal period is seen between the two periods of mania and depression. ${ }^{44}$ The behavior of those in the mania period seems to be the opposite of the behavior of the depressed. In severe periods of mania, a person is full of energy, passion, and excitement and has a lot of selfconfidence. $^{45}$

\subsection{Cytokines}

Immune system cells are widely active throughout the body, and when an infection occurs in a part of the body, these cells and their products are present at the site of infection. The process by which these results are obtained is called inflammation. Inflammation is detected in the acute phase by increasing blood flow and vascular permeability with fluid accumulation, leukocytes, and inflammatory mediators such as cytokines. In addition, lymphatic cells, inflammatory cells, and hematopoietic cells form an effective immune response. ${ }^{46}$ The complex interactions between these cells depend on a group of proteins called cytokines that act as mediators between these cells. ${ }^{47}$

Regulatory proteins are immune responses or glycoproteins that regulate target cell activity, especially in the hematopoietic system. Cytokine receptors bind to cytokines and begin sending messages and using secondary messengers in the target cell, resulting in gene activation, cell division, growth, differentiation, and migration. ${ }^{48} \mathrm{~A}$ wide range of cells produces cytokines. They are generally divided into subgroups of ILs, colony, tumor necrosis factor (TNF), interferons CSF (factor stimulator, and chemokines) because they mediate between leukocytes. Also called ILs, cytokines interact in a complex network that in various ways reduce or increase the production or function of other cytokines, and even the cytokine itself or the cytokine receptor. They have the same effects as pleiotropism and functional frequency so that the cytokine network can have a lot of flexibility. ${ }^{49}$

\subsection{Interleukins}

The acronym IL has so far been identified and is named from IL-1 to 35-IL, respectively. As the name implies, these compounds are secreted by some white blood cells and affect other white blood cells. ${ }^{44}$
IL-2 is a pro-inflammatory cytokine produced in T cells. Thus, IL-2 is an important cytokine involved in immune responses and antitumor effects. ${ }^{50}$ In addition to $\mathrm{T}$ cell secretion, natural killer (NK) cells are also involved in IL15 , with which it is aligned. Therefore, this cytokine also synergistically stimulates TNFa, IFN- $\gamma$, CSF-GM, or NK and increases cytotoxic activity. ${ }^{51}$

Studies show that internal damage molecules are released during stress-related illnesses, leading to the production of inflammatory factors, including cytokines. Therefore, it seems that during the depression, the production of cytokines is impaired. ${ }^{52}$ Cytokines are divided into two categories, inflammatory and anti-inflammatory cytokines. Inflammatory types are transcribed by NF-kB, IRF3, IRF7, and AP-1. The most important inflammatory cytokines are IL-12, IL-17, IFN- $\gamma$, TNF, IL-1, IL-2, and IL-6, and in contrast, the most important anti-inflammatory cytokines are IL-10, IL-4, and tumor growth factor- $\beta$ (TGF- $\beta) .^{53}$

\subsection{Inflammatory Cytokines in Depression Disorder}

As mentioned, inflammatory cytokines play a very important role in causing inflammation following the entry of microbes and diseases related to the immune system by activating the immune system. Because depression is a disease that is highly correlated with the immune system, many researchers have studied the pattern of inflammatory cytokines in patients with depression.

In their study 2021, Yang et al showed that serum levels of cytokines IL- 1 and IL-6 in patients with depression increased compared to the control group. Primo et al showed that the serum level of inflammatory cytokines in patients was significantly increased. ${ }^{54}$ Primo et al also suggested that anti-inflammatory drugs be used to prevent the pathophysiological effects of depression. ${ }^{55}$ Shao et al also showed that the release of inflammatory cytokines from microglial cells is significantly increased in patients with generalized depression. ${ }^{56}$ Devanda et al, in their study in 2019, also showed that IL-6 levels increase in patients with depression. ${ }^{57}$

In another review study, Farooq et al. In 2017 examined the relationship between IL-1 and the severity of depression in patients with major depression. They found that IL-1 levels were significantly increased in these patients. ${ }^{58}$

In 2020, Chapman et al showed that a variety of cytokines that increase irritable bowel disease cause depression in these patients. ${ }^{59}$

Więdłocha et al also showed that the production of inflammatory cytokines by immune cells in patients with depression is severely reduced by antidepressants. ${ }^{60}$

Köhler et al showed that IL-1, IL-2, IL-6, TNF- $a$ cytokines, the number of their products and their receptors increases in patients with depression..$^{61}$ On the other hand, in 2018, Khandaker and colleagues showed that the mentioned cytokines and the number of chemokines and intercellular adhesion molecules in the brains of depressed patients increase sharply. ${ }^{62}$ Furthermore, Kuhlman et al reported that the levels of inflammatory cytokines IL-1, TNF, and 
IFN- $\gamma$ in patients with varying degrees of depression were significantly higher than in the control group. ${ }^{63}$ Similar results from previous studies were reported in the 2019 study by Gelman et al, which showed that the expression of the inflammatory molecule TNF was significantly increased in patients with depression. ${ }^{64}$

Not all studies support an increase in the level of inflammatory cytokines in depression, and some studies have shown the opposite results. For example, Liu et al reported that serum levels of the cytokines IL- $1 \beta$, IL-8, and IL-10 did not differ in patients with major depression from those in non-depressed hospitalized patients. ${ }^{65} \mathrm{On}$ the other hand, Barry et al showed that the number of antiinflammatory cytokines such as IL-12 decreases in patients with depression. ${ }^{66}$

Such results have been identified in human studies and animal studies by various researchers in this field. In their animal study in 2020, de Sousa Tomaz et al showed that the levels of cytokines IL-10, TGF- $\beta$, and IL- 4 in the spleen and brain of depressed mice were significantly lower than in the control group. The levels of animal model cytokines in IL-6 and TNF, IL-18, and inflammatory IL-1 increase sharply. ${ }^{67}$

In another 2020 study, Ting et al cited IL-6 as a major cause of depression in depressed patients. ${ }^{68}$

As it is clear from the results of the mentioned studies, the level of inflammatory cytokines is associated with the incidence of depression in patients with depression. However, this association is not mainly related to the increase in the level of these cytokines. Some studies show an increase in inflammatory and anti-inflammatory cytokines in depression, while others show a decrease in these cytokines in depression. However, none of the mentioned studies have examined the relationship between IL-2 in patients with depression in a detailed and focused manner. In this regard, we will review the studies conducted in this field.

\subsection{Interleukin-2 Status in Major Depression}

Many studies have not examined the status of IL-2 in patients with major depression and even in patients with depression. Maes et al first reported the link between major depression and IL-2 in 1993. In their study, they justified this association by increasing the production of $\mathrm{T}$ cells following a patient suffering from depression. ${ }^{6}$

However, Maes et al showed that serum levels of IL-2 and serum levels of IL-2 receptors increase in patients with major depression. Their study evaluated patients who were in the final phase of treatment, and their results also showed an increase in serum IL-6 levels. In his study, Maes et al identified disorders in the production and secretion of IL- 6 as possible pathogenesis in major depression. ${ }^{70}$

Some studies have suggested low doses, or lower levels of IL-2, as a possible cause of major depression. Ellul et al in 2018 stated in their study that low-dose IL-2 appears to be a promising agent because it is both a stable Treg stimulator and an inhibitor of pro-inflammatory Th17 lymphocytes in humans. ${ }^{71}$

IL-2 is a therapeutic agent in a variety of cancers. However, as a therapeutic agent in cancer patients, IL-2 has side effects, like other drugs. However, the issue we are going to address here is the type of side effects of IL2. IL-2 causes symptoms are similar to depression, such as drowsiness, fatigue, loss of appetite and anorexia, irritability, and cognitive changes. In addition, Wichers et al, in their study, showed that the use of cytokines and IL-1, IL-2, and TNF- $\alpha$ affect the noradrenergic activity and cause symptoms similar to those of major depression. Finally, they stated in their study that cytokines could cause brain changes similar to those seen in depressed patients, leading to the theory that cytokines cause depression through their effects on the 5-HT, noradrenergic, and HPA systems. ${ }^{72}$

Kagaya et al evaluated several patients with major depression in Japan. The results of their study showed that plasma levels of IL-2R and TNF- $\alpha$ increased in these patients after the treatment period, and increased levels of IL-2R in plasma trigger irritability in these patients. ${ }^{73}$

Most studies show an increase in IL-2 receptor levels in depressed patients. Meanwhile, the results of Eller abd colleagues' study showed that IL-2 receptor levels in patients with major depression during treatment are different and also stated that changes in IL-2 receptor levels during treatment in these patients cause a response. ${ }^{74}$

Miller et al noted in their study that limiting or even suppressing IL-2 activity in any way improves the treatment of patients with major depression. ${ }^{75}$

In an in-vivo study, Karrenbauer et al showed that injecting IL-2 causes major depression in animals. ${ }^{76}$

Studies show that IL-2 should be increased in depressed patients with major depression and patients with other mental disorders such as schizophrenia. This is because its increase exacerbates the symptoms of mental illness, including major depression. For example, the results of a study by Al-Hakeim et al showed that IL-2 levels were higher in patients with major depression than in healthy individuals. They also stated that the level of this cytokine in patients with schizophrenia is much higher than in patients with major depression. ${ }^{77}$

In another study, Leonard et al found that IL-2 suppressants and other anti-inflammatory drugs triggered positive responses to antidepressant therapy in hospitalized patients with major depression. ${ }^{78}$

Most studies have hypothesized that the network activity of pro-inflammatory and anti-inflammatory cytokines should be considered as the pathogen of major depression. ${ }^{79,80}$

It has been proposed that dysregulated cytokine synthesis is involved in the pathogenesis and maintenance of the major depressive disorder. Cytokines are classified into proinflammatory and anti-inflammatory cytokines based on their roles. Since both pro and anti-inflammatory cytokines are altered in pathological blood and cerebrospinal fluid in major depression disorder, it has been proposed that abnormal cytokine homeostasis could be involved in the 
pathogenesis of major depression disorder and probably in developing therapeutic resistance.

\section{Discussion}

Depressive disorder is one or more severe depressive episodes in which the patient is either typically depressed or has lost interest in most activities. ${ }^{81}$ in addition, it should have at least four symptoms on the list, including changes in appetite, weight, sleep, activity, lack of energy, guilt, difficulty thinking, decision-making, and recurring thoughts of death or suicide. It should last at least two weeks. ${ }^{82}$ Depression is a term first coined in 1980 by the American Psychiatric Association to describe a set of behavioral disorders and has since become commonplace. ${ }^{83}$ Major depression leads to severe disorders in the personal, social and employment life of the affected person and affects the person's health. ${ }^{84}$ Depression has different types with different intensities, and therefore complications such as inflammation will be different in these patients. In this study, we review major depression and the inflammatory factor IL-2. In a review of published studies, we found confirmation of the association of severe depression in hospitalized patients after hospitalization. However, few studies have been performed to investigate the relationship between IL-2 levels in these patients. A limited number of studies have shown an association between IL-2 levels and the incidence of depression in hospitalized patients. In contrast, most studies have examined the relationship between other ILs in hospitalized patients.

There was no consistent pattern in the observed relationships between cytokine concentrations, changes, and clinical signs of significant depression.

Contrary to our study, Talarowska et al. showed no significant relationship between different levels of depression and the expression of cytokines IL-1, IL-6, and IL- $10 .^{85}$

In a 2009 study, Eller et al showed that over-expression of IL-2 increased response to antidepressant therapies, and there were similar results in our review. ${ }^{74}$

Clinical studies provide essential evidence that inflammation changes in a subset of patients with MDD, and articles on the role of pro-inflammatory cytokines, IL-6, in the pathophysiology of depression are increasing. For example, in Ting et al. review of IL-6 levels in the pathophysiology of depression in hospitalized patients, the results showed an association between high IL-6 levels and the incidence of major depression in hospitalized patients. ${ }^{68}$

In another study by Lanquillon et al, in 2000, results showed a significant relationship between IL-2 levels and the success rate in treating depression. In other words, the results of their study, like the results of our study, show the role of IL-2 in increasing the therapeutic response to antidepressant therapy. ${ }^{86}$

\section{Conclusion}

Finally, it can be concluded that hospitalization generally exposes the patient to inflammation. Studies show an increased risk of inflammation following hospitalization of patients, and many studies confirm the association of major depression with inflammatory cytokines and, more concentrated, IL-2. From this day on, measures should be taken to control the level of anti-inflammatory and proinflammatory cytokines in the first step and to prevent depression in the hospital in the second step, because most studies show that if the patient is not depressed, the amount of IL-2 receptors in the plasma of the hospitalized patient, the patient gradually develops depressive symptoms. One of the benefits of the present review study was to show the therapeutic properties of IL-2 in severe depression in hospitalized patients.

\section{Authors' Contributions}

All authors contributed equally to this study.

\section{Conflict of Interest Disclosures}

The authors declare that they have no conflicts of interest.

\section{Ethical Approval}

Not applicable.

\section{References}

1. Nothdurfter C, Milenkovic VM, Sarubin N, et al. The cytokine IL-17A as a marker of treatment resistance in major depressive disorder? Eur J Neurosci. 2021;53(1):172182. doi:10.1111/ejn. 14636

2. Huang KL, Chen MH, Hsu JW, Tsai SJ, Bai YM. Using classification and regression tree modeling to investigate appetite hormones and proinflammatory cytokines as biomarkers to differentiate bipolar I depression from major depressive disorder. CNS Spectr. 2021:1-7. doi:10.1017/ s109285292100016x

3. Tian $\mathrm{H}, \mathrm{Li} \mathrm{G}, \mathrm{Xu} \mathrm{G}$, et al. Inflammatory cytokines derived from peripheral blood contribute to the modified electroconvulsive therapy-induced cognitive deficits in major depressive disorder. Eur Arch Psychiatry Clin Neurosci. 2021;271(3):475-485. doi:10.1007/s00406-020-01128-9

4. Yao Y, Ju P, Liu H, et al. Ifenprodil rapidly ameliorates depressive-like behaviors, activates mTOR signaling and modulates proinflammatory cytokines in the hippocampus of CUMS rats. Psychopharmacology (Berl). 2020;237(5):14211433. doi:10.1007/s00213-020-05469-0

5. Zhan Y, Zhou Y, Zheng W, et al. Alterations of multiple peripheral inflammatory cytokine levels after repeated ketamine infusions in major depressive disorder. Transl Psychiatry. 2020;10(1):246. doi:10.1038/s41398-020-00933-Z

6. He X, Ma Q, Fan Y, et al. The role of cytokines in predicting the efficacy of acute stage treatment in patients with schizophrenia. Neuropsychiatr Dis Treat. 2020;16:191-199. doi:10.2147/ndt.s218483

7. Kim JS, Kang ES, Bahk YC, Jang S, Hong KS, Baek JH. Exploratory analysis of behavioral impulsivity, proinflammatory cytokines, and resting-state frontal EEG activity associated with non-suicidal self-injury in patients with mood disorder. Front Psychiatry. 2020;11:124. doi:10.3389/fpsyt.2020.00124

8. Himmerich H, Patsalos O, Lichtblau N, Ibrahim MAA, Dalton B. Cytokine research in depression: principles, 
challenges, and open questions. Front Psychiatry. 2019;10:30. doi:10.3389/fpsyt.2019.00030

9. Sakurai M, Yamamoto Y, Kanayama N, et al. Serum metabolic profiles of the tryptophan-kynurenine pathway in the high risk subjects of major depressive disorder. Sci Rep. 2020;10(1):1961. doi:10.1038/s41598-020-58806-w

10. Choi KW, Jang EH, Kim AY, et al. Predictive inflammatory biomarkers for change in suicidal ideation in major depressive disorder and panic disorder: a 12-week followup study. J Psychiatr Res. 2021;133:73-81. doi:10.1016/j. jpsychires.2020.12.011

11. Tonhajzerova I, Sekaninova N, Bona Olexova L, Visnovcova Z. Novel insight into neuroimmune regulatory mechanisms and biomarkers linking major depression and vascular diseases: the dilemma continues. Int J Mol Sci. 2020;21(7):2317. doi:10.3390/ijms21072317

12. Conti P, Lauritano D, Caraffa A, et al. Microglia and mast cells generate proinflammatory cytokines in the brain and worsen inflammatory state: suppressor effect of IL37. Eur J Pharmacol. 2020;875:173035. doi:10.1016/j. ejphar.2020.173035

13. Singh L, Kaur A, Garg S, Singh AP, Bhatti R. Protective effect of esculetin, natural coumarin in mice model of fibromyalgia: targeting pro-inflammatory cytokines and MAO-A. Neurochem Res. 2020;45(10):2364-2374. doi:10.1007/s11064-020-03095-y

14. Mograbi KM, Suchecki D, da Silva SG, Covolan L, Hamani C. Chronic unpredictable restraint stress increases hippocampal pro-inflammatory cytokines and decreases motivated behavior in rats. Stress. 2020;23(4):427-436. doi: 10.1080/10253890.2020.1712355

15. Tauil CB, da Rocha Lima AD, Ferrari BB, et al. Depression and anxiety in patients with multiple sclerosis treated with interferon-beta or fingolimod: role of indoleamine 2,3-dioxygenase and pro-inflammatory cytokines. Brain Behav Immun. 2020;9:100162. doi:10.1016/j. bbih.2020.100162

16. Salvador AF, de Lima KA, Kipnis J. Neuromodulation by the immune system: a focus on cytokines. Nat Rev Immunol. 2021;21(8):526-541. doi:10.1038/s41577-021-00508-Z

17. Das R, Emon MPZ, Shahriar M, et al. Higher levels of serum IL- $1 \beta$ and TNF- $\alpha$ are associated with an increased probability of major depressive disorder. Psychiatry Res. 2021;295:113568. doi:10.1016/j.psychres.2020.113568

18. Fourrier C, Baune BT. Cytokine model of cognition in relation to mental disorders during neurodevelopment. In: Perinatal Inflammation and Adult Psychopathology. Cham: Springer; 2020:253-268. doi:10.1007/978-3-030-39335-9_15

19. Kim TD, Lee S, Yoon S. Inflammation in post-traumatic stress disorder (PTSD): a review of potential correlates of PTSD with a neurological perspective. Antioxidants (Basel). 2020;9(2):107. doi:10.3390/antiox9020107

20. Toft H. Cytokines and Psychiatric Symptoms in Patients Receiving Inpatient Treatment: The Relationship Between Changes in Immune Activation and Symptoms of Mental Distress: A 12-Week Follow-Up Study of Patients with Mental Health Disorders. Norway: University of Oslo; 2020.

21. Liu Z, Mao X, Dan Z, et al. Gene variations in autism spectrum disorder are associated with alteration of gut microbiota, metabolites and cytokines. Gut Microbes. 2021;13(1):1-16. doi:10.1080/19490976.2020.1854967

22. Mansur RB, Delgado-Peraza F, Subramaniapillai M, et al. Extracellular vesicle biomarkers reveal inhibition of neuroinflammation by infliximab in association with antidepressant response in adults with bipolar depression. Cells. 2020;9(4):895. doi:10.3390/cells9040895

23. Coryell W, Wilcox H, Evans SJ, et al. Latent infection, inflammatory markers and suicide attempt history in depressive disorders. J Affect Disord. 2020;270:97-101. doi:10.1016/j.jad.2020.03.057

24. Böttcher C, Fernández-Zapata C, Snijders GJL, et al. Singlecell mass cytometry of microglia in major depressive disorder reveals a non-inflammatory phenotype with increased homeostatic marker expression. Transl Psychiatry. 2020;10(1):310. doi:10.1038/s41398-020-00992-2

25. Guglielminotti J, Li G. Exposure to general anesthesia for cesarean delivery and odds of severe postpartum depression requiring hospitalization. Anesth Analg. 2020;131(5):14211429. doi: 10.1213 /ane. 000000000004663

26. Sade S, Sheiner E, Wainstock T, et al. Risk for depressive symptoms among hospitalized women in high-risk pregnancy units during the COVID-19 pandemic. J Clin Med. 2020;9(8):2449. doi:10.3390/jcm9082449

27. Cepeda MS, Schuemie M, Kern DM, Reps J, Canuso C. Frequency of rehospitalization after hospitalization for suicidal ideation or suicidal behavior in patients with depression. Psychiatry Res. 2020;285:112810. doi:10.1016/j. psychres.2020.112810

28. Cliffe C, Shetty H, Himmerich H, Schmidt U, Stewart R, Dutta R. Suicide attempts requiring hospitalization in patients with eating disorders: a retrospective cohort study. Int J Eat Disord. 2020;53(5):458-465. doi:10.1002/eat.23240

29. Gialluisi A, Costanzo S, Castelnuovo AD, et al. Combined influence of depression severity and low-grade inflammation on incident hospitalization and mortality risk in Italian adults. J Affect Disord. 2021;279:173-182. doi:10.1016/j. jad.2020.10.004

30. Bättig VAD, Roll SC, Hahn M. Pharmacogenetic testing in depressed patients and interdisciplinary exchange between a pharmacist and psychiatrists results in reduced hospitalization times. Pharmacopsychiatry. 2020;53(4):185192. doi:10.1055/a-1096-1171

31. Peters EM, Dong LY, Thomas T, et al. Instability of suicidal ideation in patients hospitalized for depression: an exploratory study using smartphone ecological momentary assessment. Arch Suicide Res. 2020:1-14. doi:10.1080/13811 118.2020.1783410

32. Salvatore P, Khalsa HK, Tohen M, Baldessarini RJ. Long-term morbidity in major affective and schizoaffective disorders following hospitalization in first psychotic episodes. Acta Psychiatr Scand. 2021;143(1):50-60. doi:10.1111/acps. 13243

33. Edgcomb JB, Thiruvalluru R, Pathak J, Brooks JO 3rd. Machine learning to differentiate risk of suicide attempt and self-harm after general medical hospitalization of women with mental illness. Med Care. 2021;59:S58-S64. doi:10.1097/ mlr.0000000000001467

34. Eisenberg MJ, Habib B, Alcaraz M, Thombs BD, Filion KB. Bright light therapy for depressive symptoms in hospitalized cardiac patients: a randomized controlled pilot trial. PLoS One. 2020;15(3):e0230839. doi:10.1371/journal. pone.0230839

35. Zahid S, Bodicherla KP, Eskander N, Patel RS. Attentiondeficit/hyperactivity disorder and suicidal risk in major depression: analysis of 141,530 adolescent hospitalizations. 
Cureus. 2020;12(5):e7949. doi:10.7759/cureus.7949

36. Adjei Boakye E, Osazuwa-Peters N, Mohammed KA, et al. Prevalence and factors associated with diagnosed depression among hospitalized cancer patients with metastatic disease. Soc Psychiatry Psychiatr Epidemiol. 2020;55(1):15-23. doi:10.1007/s00127-019-01763-1

37. Aouidad A, Cohen D, Mirkovic B, et al. Borderline personality disorder and prior suicide attempts define a severity gradient among hospitalized adolescent suicide attempters. BMC Psychiatry. 2020;20(1):525. doi:10.1186/ s12888-020-02930-4

38. Geng F, Jiang F, Conrad R, et al. Factors associated with involuntary psychiatric hospitalization of youths in China based on a nationally representative sample. Front Psychiatry. 2020;11:607464. doi:10.3389/fpsyt.2020.607464

39. Kany S, Vollrath JT, Relja B. Cytokines in inflammatory disease. Int J Mol Sci. 2019;20(23):6008. doi:10.3390/ ijms 20236008

40. Zhou WJ, Yang HL, Shao J, et al. Anti-inflammatory cytokines in endometriosis. Cell Mol Life Sci. 2019;76(11):2111-2132. doi:10.1007/s00018-019-03056-x

41. Chen Z, Bozec A, Ramming A, Schett G. Anti-inflammatory and immune-regulatory cytokines in rheumatoid arthritis. Nat Rev Rheumatol. 2019;15(1):9-17. doi:10.1038/s41584018-0109-2

42. Saghazadeh A, Ataeinia B, Keynejad K, Abdolalizadeh A, Hirbod-Mobarakeh A, Rezaei N. A meta-analysis of proinflammatory cytokines in autism spectrum disorders: effects of age, gender, and latitude. J Psychiatr Res. 2019;115:90-102. doi:10.1016/j.jpsychires.2019.05.019

43. Aggarwal R, Jain AK, Mittal P, Kohli M, Jawanjal P, Rath G. Association of pro- and anti-inflammatory cytokines in preeclampsia. J Clin Lab Anal. 2019;33(4):e22834. doi:10.1002/jcla.22834

44. Jenny NS, Callas PW, Judd SE, et al. Inflammatory cytokines and ischemic stroke risk: the REGARDS cohort. Neurology. 2019;92(20):e2375-e2384. doi:10.1212/ wnl.0000000000007416

45. Miranda TS, Heluy SL, Cruz DF, et al. The ratios of proinflammatory to anti-inflammatory cytokines in the serum of chronic periodontitis patients with and without type 2 diabetes and/or smoking habit. Clin Oral Investig. 2019;23(2):641-650. doi:10.1007/s00784-018-2471-5

46. Taipa R, das Neves SP, Sousa AL, et al. Proinflammatory and anti-inflammatory cytokines in the CSF of patients with Alzheimer's disease and their correlation with cognitive decline. Neurobiol Aging. 2019;76:125-132. doi:10.1016/j. neurobiolaging.2018.12.019

47. He Y, Li W, Wang Y, et al. Major depression accompanied with inflammation and multiple cytokines alterations: evidences from clinical patients to Macaca fascicularis and LPS-induced depressive mice model. J Affect Disord. 2020;271:262-271. doi:10.1016/j.jad.2020.03.131

48. Soare AY, Durham ND, Gopal R, et al. P2X antagonists inhibit HIV-1 productive infection and inflammatory cytokines interleukin-10 (IL-10) and IL-1 $\beta$ in a human tonsil explant model. J Virol. 2019;93(1):e01186-18. doi:10.1128/ jvi.01186-18

49. Gallenga CE, Pandolfi F, Caraffa A, et al. Interleukin-1 family cytokines and mast cells: activation and inhibition. $J$ Biol Regul Homeost Agents. 2019;33(1):1-6.

50. Gupta KK, Khan MA, Singh SK. Constitutive inflammatory cytokine storm: a major threat to human health. J Interferon Cytokine Res. 2020;40(1):19-23. doi:10.1089/jir.2019.0085

51. Gholijani N, Yazdani MR, Dastgheib L. Predominant role of innate pro-inflammatory cytokines in vitiligo disease. Arch Dermatol Res. 2020;312(2):123-131. doi:10.1007/s00403019-01996-9

52. Zhou WJ, Yang HL, Shao J, et al. Anti-inflammatory cytokines in endometriosis. Cell Mol Life Sci. 2019;76(11):2111-2132. doi:10.1007/s00018-019-03056-X

53. Pawluk H, Woźniak A, Grześk G, et al. The role of selected pro-inflammatory cytokines in pathogenesis of ischemic stroke. Clin Interv Aging. 2020;15:469-484. doi:10.2147/cia. s233909

54. Yang KC, Liu MN, Liou YJ, Hu LY, Yang BH, Chou YH. Interleukin-1 family and serotonin transporter in firstepisode, drug-naive major depressive disorder: a pilot study. J Psychiatr Res. 2021;135:174-180. doi:10.1016/j. jpsychires.2021.01.018

55. de Carvalho Alves LP, da Rocha NS. Different cytokine patterns associate with melancholia severity among inpatients with major depressive disorder. Ther Adv Psychopharmacol. 2020;10:2045125320937921. doi:10.1177/2045125320937921

56. Shao SL, Liu DQ, Zhang B, Huang SM. Inflammatory response, a key pathophysiological mechanism of obesityinduced depression. Mediators Inflamm. 2020;2020:8893892. doi:10.1155/2020/8893892

57. Devanda R, Sihag PM. Status of interluekin-6 and lipid profile in depression disorder. Int J Clin Biochem Res. 2019;6(2):230-233. doi:10.18231/j.ijcbr.2019.051

58. Farooq RK, Asghar K, Kanwal S, Zulqernain A. Role of inflammatory cytokines in depression: focus on interleukin1ß. Biomed Rep. 2017;6(1):15-20. doi:10.3892/br.2016.807

59. Chapman BJ, Jones GB. That gut feeling: the role of inflammatory cytokines in depression among patients with inflammatory bowel disease. EMJ Gastroenterol. 2020;9(1):81-90.

60. Więdłocha M, Marcinowicz P, Krupa R, et al. Effect of antidepressant treatment on peripheral inflammation markers - a meta-analysis. Prog Neuropsychopharmacol Biol Psychiatry. 2018;80(Pt C):217-226. doi:10.1016/j. pnpbp.2017.04.026

61. Köhler CA, Freitas TH, Stubbs B, et al. Peripheral alterations in cytokine and chemokine levels after antidepressant drug treatment for major depressive disorder: systematic review and meta-analysis. Mol Neurobiol. 2018;55(5):4195-4206. doi:10.1007/s12035-017-0632-1

62. Khandaker GM, Oltean BP, Kaser M, et al. Protocol for the insight study: a randomised controlled trial of single-dose tocilizumab in patients with depression and low-grade inflammation. BMJ Open. 2018;8(9):e025333. doi:10.1136/ bmjopen-2018-025333

63. Kuhlman KR, Robles TF, Dooley LN, Boyle CC, Haydon MD, Bower JE. Within-subject associations between inflammation and features of depression: Using the flu vaccine as a mild inflammatory stimulus. Brain Behav Immun. 2018;69:540-547. doi:10.1016/j.bbi.2018.02.001

64. Leff Gelman P, Mancilla-Herrera I, Flores-Ramos M, et al. The cytokine profile of women with severe anxiety and depression during pregnancy. BMC Psychiatry. 2019;19(1):104. doi:10.1186/s12888-019-2087-6

65. Liu JJ, Wei YB, Strawbridge R, et al. Peripheral cytokine levels 
and response to antidepressant treatment in depression: a systematic review and meta-analysis. Mol Psychiatry. 2020;25(2):339-350. doi:10.1038/s41380-019-0474-5

66. Barry A, O'Halloran KD, McKenna JP, McCreary C, Downer EJ. Plasma IL-8 signature correlates with pain and depressive symptomatology in patients with burning mouth syndrome: results from a pilot study. J Oral Pathol Med. 2018;47(2):158165. doi:10.1111/jop. 12666

67. de Sousa Tomaz V, Chaves Filho AJ, Cordeiro RC, et al. Antidepressants of different classes cause distinct behavioral and brain pro- and anti-inflammatory changes in mice submitted to an inflammatory model of depression. J Affect Disord. 2020;268:188-200. doi:10.1016/j.jad.2020.03.022

68. Ting EY, Yang AC, Tsai SJ. Role of interleukin-6 in depressive disorder. Int J Mol Sci. 2020;21(6):2194. doi:10.3390/ ijms21062194

69. Maes M, Stevens WJ, Declerck LS, et al. Significantly increased expression of T-cell activation markers (interleukin-2 and HLA-DR) in depression: further evidence for an inflammatory process during that illness. Prog Neuropsychopharmacol Biol Psychiatry. 1993;17(2):241255. doi:10.1016/0278-5846(93)90045-t

70. Maes M, Meltzer HY, Bosmans E, et al. Increased plasma concentrations of interleukin-6, soluble interleukin-6, soluble interleukin-2 and transferrin receptor in major depression. J Affect Disord. 1995;34(4):301-309. doi:10.1016/0165-0327(95)00028-1

71. Ellul P, Mariotti-Ferrandiz E, Leboyer M, Klatzmann D. Regulatory T cells as supporters of psychoimmune resilience: toward immunotherapy of major depressive disorder. Front Neurol. 2018;9:167. doi:10.3389/fneur.2018.00167

72. Wichers M, Maes M. The psychoneuroimmunopathophysiology of cytokine-induced depression in humans. Int J Neuropsychopharmacol. 2002;5(4):375-388. doi:10.1017/s1461145702003103

73. Kagaya A, Kugaya A, Takebayashi M, et al. Plasma concentrations of interleukin-1beta, interleukin-6, soluble interleukin-2 receptor and tumor necrosis factor alpha of depressed patients in Japan. Neuropsychobiology. 2001;43(2):59-62. doi:10.1159/000054867

74. Eller T, Vasar V, Shlik J, Maron E. Pro-inflammatory cytokines and treatment response to escitalopram in major depressive disorder. Prog Neuropsychopharmacol Biol Psychiatry. 2008;32(2):445-450. doi:10.1016/j.pnpbp.2007.09.015

75. Miller AH, Maletic V, Raison CL. Inflammation and its discontents: the role of cytokines in the pathophysiology of major depression. Biol Psychiatry. 2009;65(9):732-741. doi:10.1016/j.biopsych.2008.11.029

76. Karrenbauer BD, Müller CP, Ho YJ, et al. Time-dependent in-vivo effects of interleukin-2 on neurotransmitters in various cortices: relationships with depressive-related and anxiety-like behaviour. J Neuroimmunol. 2011;237(1-2):2332. doi:10.1016/j.jneuroim.2011.05.011

77. Al-Hakeim HK, Al-Rammahi DA, Al-Dujaili AH. IL-6, IL-18, sIL-2R, and TNFa proinflammatory markers in depression and schizophrenia patients who are free of overt inflammation. J Affect Disord. 2015;182:106-114. doi:10.1016/j.jad.2015.04.044

78. Leonard BE. Inflammation and depression: a causal or coincidental link to the pathophysiology? Acta Neuropsychiatr. 2018;30(1):1-16. doi:10.1017/neu.2016.69

79. Petralia MC, Mazzon E, Fagone P, et al. The cytokine network in the pathogenesis of major depressive disorder. Close to translation? Autoimmun Rev. 2020;19(5):102504. doi:10.1016/j.autrev.2020.102504

80. Zhou B, Zhu Z, Ransom BR, Tong X. Oligodendrocyte lineage cells and depression. Mol Psychiatry. 2021;26(1):103117. doi:10.1038/s41380-020-00930-0

81. Troubat R, Barone P, Leman S, et al. Neuroinflammation and depression: a review. Eur J Neurosci. 2021;53(1):151-171. doi:10.1111/ejn.14720

82. Petralia MC, Mazzon E, Fagone P, et al. Pathogenic contribution of the macrophage migration inhibitory factor family to major depressive disorder and emerging tailored therapeutic approaches. J Affect Disord. 2020;263:15-24. doi:10.1016/j.jad.2019.11.127

83. Petrozzi BP, Blecker C, Neumann E, Sammer G. Hypothalamic Glutamine-Glutamate Metabolites and Peripheral Pro-Inflammatory Cytokines in Participants with Depression: A Case-Control Study. Research Square; 2020. doi:10.21203/rs.3.rs-105917/v1

84. Tucker JA, Osann K, Hsieh S, et al. Longitudinal changes in sleep: associations with shifts in circulating cytokines and emotional distress in a cancer survivor population. Int $J$ Behav Med. 2021;28(1):140-150. doi:10.1007/s12529-02009950-0

85. Talarowska M, Szemraj J, Gałecki P. The role of interleukin genes in the course of depression. Open Med (Wars). 2016;11(1):41-48. doi:10.1515/med-2016-0009

86. Lanquillon S, Krieg JC, Bening-Abu-Shach U, Vedder $\mathrm{H}$. Cytokine production and treatment response in major depressive disorder. Neuropsychopharmacology. 2000;22(4):370-379. doi:10.1016/s0893-133x(99)00134-7 